\title{
The XXXVII European muscle conference: Oxford September 2008
}

\author{
Sanford I. Bernstein · Michael A. Geeves
}

Received: 24 October 2008/Accepted: 30 October 2008/Published online: 26 November 2008

(C) Springer Science+Business Media B.V. 2008

The 37th European Muscle Conference was held at Keble College, Oxford, UK from the 13th through 16th of September 2008. Steven Marston (Imperial College, London) and Charles Redwood (Oxford) organized a diverse and high quality program. Despite an extremely rainy late summer in England, the elements cooperated to provide pleasant weather for conference delegates to enjoy Oxford. Festivities included an outdoor champagne reception. This was followed by a gala dinner held in a college dining hall reminiscent of the one used in the Harry Potter films. Multiple poster sessions complemented an intensive series of oral presentations. Topics covered included thin and thick filaments, the muscle cytoskeleton, myosin motors, muscle development and plasticity, skeletal, smooth and cardiac muscle contractility, muscle disease and therapies, and muscle genomics and proteomics.

Outstanding young investigators were recognized at the conference for the best oral and poster presentations. Oral presentation awards went to Katja Gehmlich (University College, London), Sabine van Dijk (VU Medical Center, The Netherlands), Pedro Velica (University of Birmingham) and Clare Gallon (Imperial College, London). The young investigator poster presentation awards went to Ilya Nevzorov (Royal Academy of Sciences, Moscow) and Jaakko Sarparanta (University of Helsinki), with Christiane Look (Max-Delbrueck-Center for Molecular Medicine, Berlin) and Zacharias Orfanos (University of York)

S. I. Bernstein $(\square)$

Department of Biology, Molecular Biology Institute, Heart Institute, San Diego State University, San Diego, CA, USA e-mail: sbernst@sciences.sdsu.edu

M. A. Geeves

Department of Biosciences, University of Kent, Kent, UK

e-mail: m.a.geeves@kent.ac.uk receiving awards sponsored by the Lymn Foundation. The judges for the young investigator awards were Anders Arner, Carol Gregorio, Ger Steinen, Geoff Walker, Sacha Vorotnikov, Laurence Stevens, and Dominic Wells. The abstracts for the awardees and for other participants in the conference can be found below.

\section{Through thin and thick}

Many presentations focused on the structure of thin and thick filaments, cross-bridge function and details of myosin motor mechanics. Bill Lehman (Boston University School of Medicine) started the conference off with a remarkably large audience for 8:30 a.m. on a Sunday morning. This was a tribute to the organizers of the conference and the attraction of listening to Lehman's talk on tropomyosin. He covered the background to the recent electron microscopic work on identifying the different positions of tromomyosin on actin: the blocked or B state, which covers most of the myosin binding site on actin, the closed, calcium induced or C-state, which only allows weak binding of myosin and the myosin-induced or $\mathrm{M}$ state which allows full productive interaction with myosin. He provided new insights from labelling actin and actin tropomyosin filaments with a C-terminal peptide of troponin I that decorates the N-terminus of actin. This peptide competes with the tropomyosin for the $\mathrm{C}$-position on actin thus favoring the $\mathrm{B}$ state of tropomyosin on actin. The peptide also appears to interact with tropomyosin itself. The degree of flexibility in tropomyosin and what is meant by flexibility has been hotly debated in recent years. Lehman presented recent molecular dynamics modeling data indicating uniform motions of tropomyosin along its length that are of the right order to allow tropomyosin to stiffen the actin filament yet 
move over the surface of actin between the three defined positions.

Belinda Bullard (University of York) presented work on the unusual forms of troponin $\mathrm{C}$ found in insect flight muscle. Two isoforms are present; one (TnC-F1) with a single calcium binding site in the C-terminal "structural domain" appears responsible for the calcium activation of oscillatory activity, the other (TnC-F2) with a single calcium binding site in each of the "structural" and "regulatory" domains appears to activate isometric contraction. The open/closed transition of the calcium binding domains does not appear altered in NMR structures on calcium binding. How these $\mathrm{TnC}$ isoforms regulate muscle activity, therefore remains an intriguing mystery.

Some lessons learned from non-muscle myosins were particularly fascinating. The light chain binding regions of muscle and some non-muscle myosins are well known to serve as lever arms that rotate during the power stroke. However, Peter Knight (University of Leeds) reported that unconventional myosins 6, 7a, 10 and MyoM possess single alpha-helix (SAH) domains that extend the length of the lever. Although originally predicted to form alpha-helical coiled-coils with a second heavy chain, linear SAH domains can be observed by metal shadowing in the electron microscope. TIRF measurements of GFP-tagged molecules indicate that a SAH domain increases the step size of the myosin molecule on the actin filament, likely by serving as a stiff element that amplifies the lever arm rotation.

Balint Kintses (Eötvös University, Budapest) reported on the mechanism of ATPase activation by actin in myosin II of the slime mold Dictyostelium. Interestingly, kinetic studies that monitored a single tryptophan on the relay helix, which is coupled to both the SWII Pi sensor and the converter domain, showed that the rate-limiting step of this myosin's basal ATPase is the "reverse recovery stroke" of the lever arm. This is the equivalent of the power stroke, had the molecule been attached to actin. This may be the conformational change that limits the $\mathrm{Pi}$ release from M.ADP.Pi, which has previously been postulated to limit myosin's basal ATPase cycle. Computer simulations performed by Balint et al. suggest that actin binding is communicated directly to the relay helix through a prolinerich loop. The resulting movement of the relay helix ultimately induces the reverse recovery (power) stroke. In agreement with the computer simulations, mutating the myosin to remove the proline-rich loop disables actin activation of the reverse recovery stroke. It will be interesting to determine whether actin activates the myosin ATPase by the same mechanism in muscle myosins.

Mihaly Kovacs (Eötvös University, Budapest) provided new insights into the blebbistatin inhibitor of myosin II. This is known to bind in the actin binding cleft thereby trapping the ADP.Pi complex and inhibiting Pi release from both myosin and actomyosin. The new data suggest that a M.ADP complex with blebbistatin can bind to actin and may present a form of actomyosin so far inaccessible to structural studies. It will be fascinating to see if this complex can be analyzed at high resolution.

There was a clear clash of opinion between John Squire (University of Bristol) and Malcolm Irving (King's College, London) on the interpretation of X-ray scattering data from muscle fibers, in particular the interpretation of the meridional $14.3 \mathrm{~nm}$, M3 X-ray refection. The Irving group has interpreted this to give unprecedented insights into the movement of myosin cross-bridges following mechanical activity by analyzing the way the interference between the two halves of the sarcomere alter the fine splitting of the M3 reflection. Irving gave an impressive analysis of the data indicating that the cross-bridges maintain $6 \mathrm{pN}$ of force through a $6 \mathrm{~nm}$ stoke and discussed the implications of this for how we think about the working cycle of the cross-bridge. Squire in contrast provided an argument that the M3 reflection during a mechanical transient does not contain sufficient information to interpret how the cross bridges are moving. This appears to be such a fundamental difference of views that it should be possible to resolve itbut it did not occur on this occasion.

\section{In sickness and in health}

Caroline Sewry (Institute of Child Health, London) provided insights into the clinical and pathological defects associated with congenital myopathies. Not only can different genes cause diseases that fall under the same classification, different mutations in one gene can cause different diseases. Thus accurate diagnosis and treatment are challenging prospects. Nigel Laing (University of Western Australia) gave an overview of skeletal muscle disease caused by mutant sarcomeric proteins. These include various myopathies caused by mutations in different myosin heavy chain genes and nemaline myopathies that derive from mutations in thin filament associated proteins. Nemaline myopathies generally are non-progressive and biopsies show nemaline bodies of various shapes (often elongated or thread-like). These diseases can arise from mutations in nebulin, alpha and beta tropomyosins, actin, cofilin 2 or troponin T. Some humans with nemaline myopathy are homozygous null for skeletal muscle actin but are able to survive by retaining expression of the cardiac muscle actin gene in skeletal muscles. Interestingly, Laing reported that mice expressing the cardiac muscle actin gene in place of the skeletal muscle gene show normal muscle structure and function, suggesting that further up-regulating cardiac actin genes in human skeletal muscle actin nulls could prove an effective therapy. In contrast, Copeland and colleagues (Imperial College, 
London) presented a poster showing that skeletal muscle actin is switched on at high levels in humans suffering from end-stage failure and hypertrophic obstructive cardiomyopathy. It is interesting to speculate whether this is a compensatory mechanism that relies upon increasing the overall levels of actin or if the subtle differences in sequence of the skeletal muscle actin isoform are critical for compensation.

Lars Larsson (Uppsala University) talked about myosin loss and muscle paralysis that is sometimes observed in intensive care unit patients. This syndrome, known as acute quadriplegic myopathy is the most common type of acquired myopathy and is often correlated with ventilator use and administration of steroids. Larsson has developed porcine and rodent models of acute quadriplegic myopathy. Both transcriptome and proteome studies are being carried out in order to understand the molecular basis of thick filament loss.

Exercise, while generally considered beneficial to muscle function, can also lead to myofibrillar disarray and relocalization of chaperones of the small heat shock family, according to Frederik Lauritzen (University of Oslo). Human volunteers performed eccentric exercise, wherein contracting muscle is forcibly stretched, and provided biopsy samples for electron microscopic examination. Abnormalities observed included Z-disk streaming, disarrayed myofibrils, centrally located nuclei and immune cell infiltration. Immunogold labeling of the small heat shock proteins $\alpha \mathrm{B}$-crystallin and Hsp-27 showed predominantly cytoplasmic localization in control muscles. After eccentric exercise, the proteins relocalized to the myofibrils, particularly at the Z-disks. In severely disrupted areas of the myofibrils the $\alpha \mathrm{B}$-crystallin tended to preferentially accumulate at disrupted Z-disks, whereas the Hsp-27 was less specifically localized. Evidence for an in vivo role of Hsp27 can be inferred from the in vitro studies presented by Nikolai Gusev (Moscow State University). He reported that Hsp-27 is capable of preventing aggregation of heated myosin or actin. By relocalizing to myofibrils in vivo, Hsp27 may prevent contractile protein aggregation in the cell.

\section{Taking it to heart}

Hugh Watkins (Oxford) provided an excellent overview of inherited cardiomyopathies caused by contractile protein mutations. It is becoming clear that mutations resulting in dilated cardiomyopathy typically reduce the calcium sensitivity of muscle contraction, diminish myosin ATPase activity and/or decrease in vitro motility of thin filaments. Mutations causing hypertrophic cardiomyopathy generally have the opposite effects. Watkins sounded a cautionary note regarding mapping of disease-causing mutations by sequencing candidate genes. If a mutation in a candidate gene is found, one should not conclude that the mutation causes the disease, as it could instead be an unrelated polymorphism. One way to be fairly certain that the mutation is causative, is if it occurs in multiple families with the same disease. Another strong indication is when the mutation arises de novo, i.e., it is not present in either parent. Absent these observations, a careful genetic segregation analysis is imperative as well as demonstration that the identified mutation does not occur in ethnically matched individuals who lack the disease. Dr. Watkins illustrated his concern by citing several studies in high profile journals that, in retrospect, wrongly identified cardiomyopathy mutations. This caution must extend to biochemists and those making animal models, who should carefully scrutinize the clinical results to define mutant proteins worth studying.

A number of studies presented at the meeting investigated the physiological and biochemical defects associated with cardiomyopathies. Jil Tardiff (Albert Einstein College of Medicine, New York) reported on troponin T, Bogdan Iorga (University of Cologne) on troponin I, Weihua Song (Imperial College London) on cardiac actin, Katja Gehmlich (University College London) and Pauline Bennett (King's College London) on muscle LIM protein, and Sabine van Dijk (VU Medical Center, The Netherlands) and Alexandra Belus (University of Florence) on myosin binding protein-C. Sanford Bernstein (San Diego State University) discussed the Drosophila heart as a model system for investigating cardiomyopathies, reporting that myosin heavy chain mutations that cause dilated or restrictive phenotypes arise from mutations that down- or up-regulate myosin function, respectively.

Several speakers discussed the roles of protein phosphorylation in myocardial contractility and disease. John Solaro (University of Illinois at Chicago) reported on p21 activated kinase's ability to stimulate the myofilament $\mathrm{Ca}^{2+}$ response. Martina Krüger (University of Münster) explained that phosphorylation by cyclic GMP dependent protein kinase reduces titin persistence length, which results in decreased passive fiber stiffness. Viola Kooij (VU Medical Center, The Netherlands) documented protein kinase $\mathrm{C}-\alpha$ phosphorylation of troponin $\mathrm{T}$, troponin $\mathrm{I}$ and other myocardial proteins in failing myocardium and concluded that this results in reduced $\mathrm{Ca}^{2+}$ sensitivity of force development. Aaron Hinken (University of Illinois at Chicago) discussed how $\mathrm{PKC} \delta$-dependent myofilament phosphorylation requires phosphorylation of $\operatorname{PKC} \delta$ itself. Finally, Jennifer van Eyk (Johns Hopkins University, Baltimore) more broadly explored post-translational modifications of mitochondrial proteins using a proteomics approach. She showed that major changes in post-translational modifications are associated with the myocardium in heart failure. 


\section{Developing stories}

Assembly of the various contractile proteins into a myofibril is a fascinating process that is far from understood. Two giant proteins of the vertebrate sarcomere, titin and nebulin, appear to play key roles. Titin stretches from the M-band and connects the thick filaments to the Z-disk, whereas nebulin extends from the thin filaments to the Z-disk. Carol Gregorio (University of Arizona, Tucson) reported that nebulin interacts with the barbed-end actin capping protein CapZ, which is a Z-disk component. Interestingly, data suggest that nebulin binds to CapZ from an actin filament of an adjacent sarcomere. This could serve to link the sarcomeres and reinforce the integrity of the myofibril. Mathias Gautel (King's College, London) presented interesting mechanical data on how strain might activate the titin kinase domain by allowing ATP binding that then triggers autophosphorylation and further kinase activity. The kinase domain is thus a biological force sensor that can contribute to adaptation of muscle to changes in loading. Elisabeth Ehler (King's College, London) suggests that during development, titin may work with the M-band proteins and the Z-disk protein $\alpha$-actinin to constitute a "sarcomeroskeleton" onto which additional proteins are assembled. She reported that the first complexes assembled during sarcomere development are composed of the N-terminus of titin, $\alpha$-actinin and actin. Further, myomesin, an M-band protein, is always associated with assembling thick filaments. For thick filament assembly in Drosophila indirect flight muscle, John Sparrow (University of York) reported that one myosin heavy chain isoform initiates the process and a second completes it. Perhaps the initiator myosin has affinity for the Drosophila M-band complex and serves to localize the thick filament appropriately.

The European Muscle Conference in 2009 will be held in Lille, France from the 12th through the 16th of September. Based upon the quality of this year's meeting, we expect a good turnout! 